\title{
Fermilab
}

Do Engineering Note

3740.222-EN-60

September 23, 1986

\section{Forces on the Middle EC Module Connections \\ Resulting From Assembly Sequence A}

Bob Wands

\section{Introduction}

The ANSYS superelement model representing the sequential assembly of the ec modules has been used to calculate the maximum forces experienced at any interface between middle modules during assembly. The assembly sequence used was devised by GTM and is referred to as Sequence $A$. (see attached sheet for explanation of sequence).

Fig. 1 shows the assembled model and support. One-half symmetry is assumed. The superelement model supported the OCH modules at theta $=56.25$ in such a way that the support was allowed to translate freely in the horizontal direction, and rotate freely about the longitudinal (beam) axis. This produces "worst case" module connecting forces, and is justified given the importance of these connections. The middle modules were supported within the $\mathrm{OCH}$ arch only through theta $=56.25$.

Each middle module interface contained six connection points in the finite element model through which the forces were transmitted between modules. (see Fig. 2). In presenting the results here, the forces at the three connection locations at the inner radius of each interface were summed, and the 
largest value found. This was also done for the outer radius. In applying these results to the design of the actual connections, the forces presented here should be divided by the actual number of connection points used.

$\underline{\text { Results }}$

The force normal to each interface (FN) and the force parallel to each interface (FS) as well as the resolved force on the inner and outer locations was calculated and is included here as Appendix A.

The resulting maximum connecting forces for the inner and outer radii are:

Inner Radius: The maximum force at the inner radius is 35000 lbs, and occurs at theta=33.75 for Step 30 of Assembly Sequence A. (Step 30 is the fully assembled structure)

Outer Radius: The maximum force at the outer radius is $48000 \mathrm{lbx}$. and occurs at theta=33.75 for Step 30 of Assembly Sequence A. (Step 30 is the fully assembled structure)

\section{Conclusion}

The maximum loads occur when the EC modules (including the IFH) are fully assembled. No larger loads occur during the assembly process. The numbers presented here can be applied to the actual connection design by remembering that they represent the total force at each radius, and must be divided by the number of actual connection locations used. For example, if two connections are used at the inner and outer radii (for a total of four locations) the maximum load for a single connection is $48000 / 2$ or 24000 lbs. 


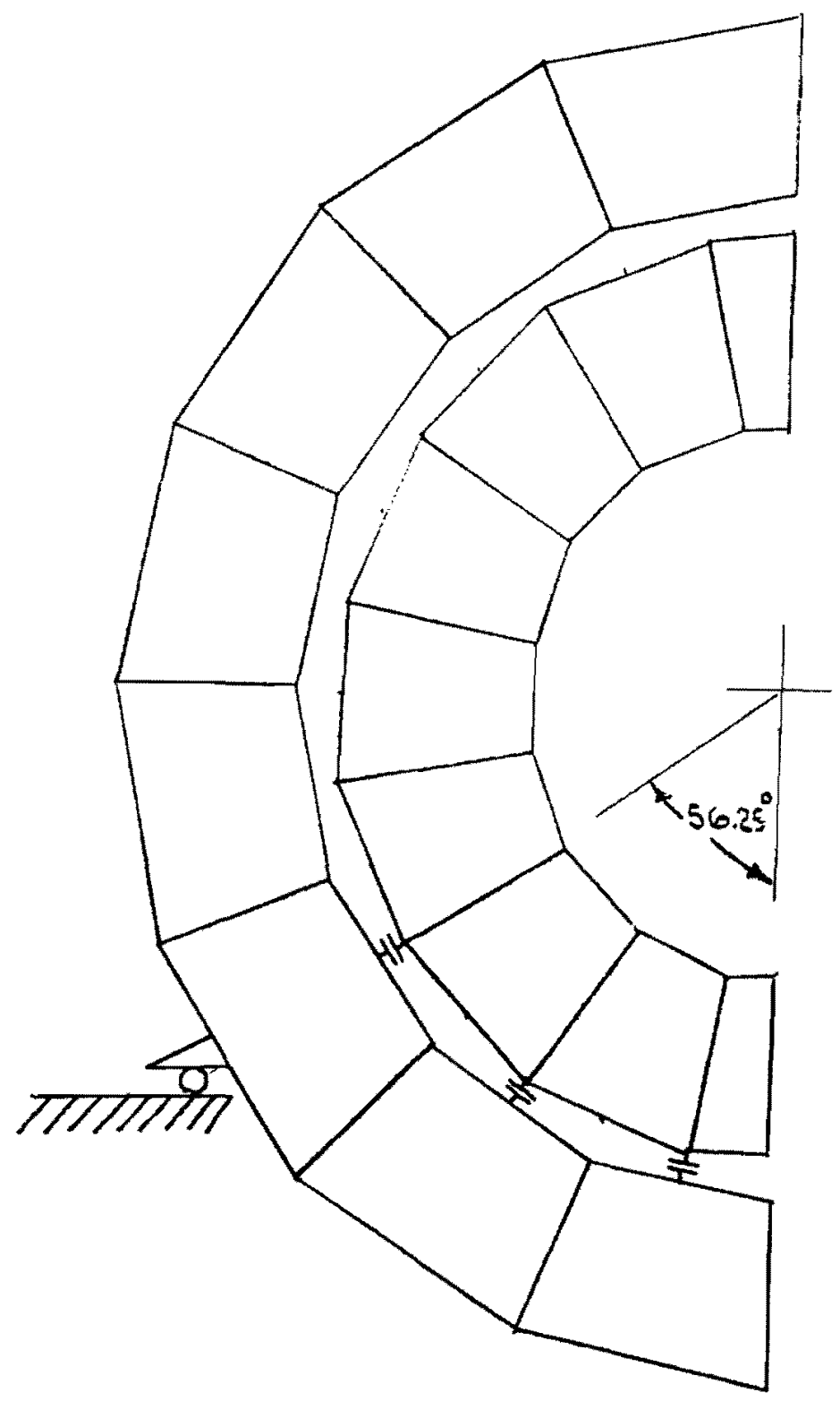

Fig 1. Schematic of EC Superelement Model (Shown fully assembled) 


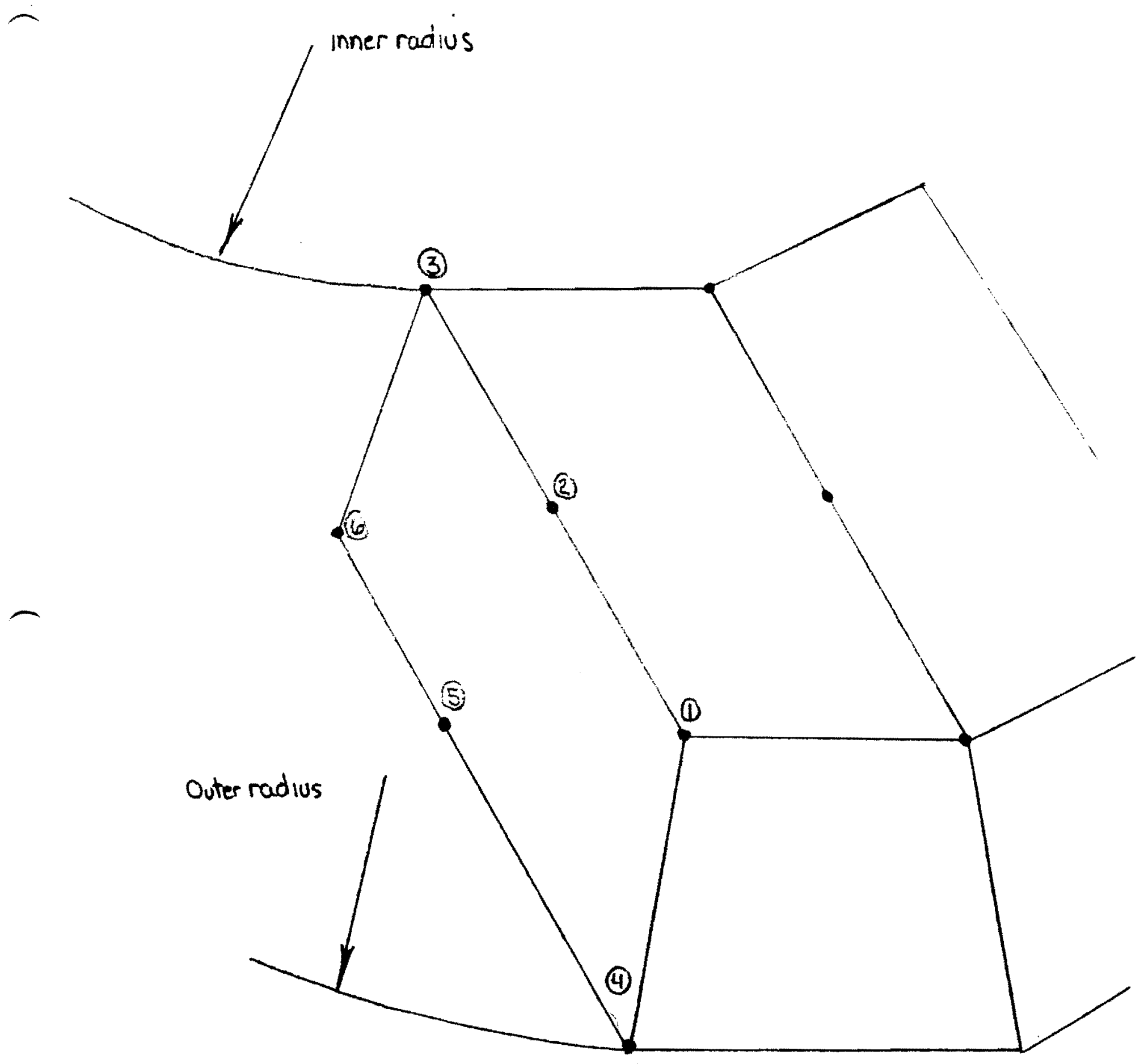

Fig 2. Location of the six connection Points at a typical EC middle module interface 
Anguat 23,1936

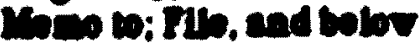

B: DC Yodulo

Asmoly Soquence

Froa; G.T Wulholland

It has long been recognized the aceombly squence of the $X C$ motules into the cryostat may croate larger inter-modular stresses or lateral reactions then the fulty assembled calorimeter. Guided by those things ve heve loarned, a sense of symmotry. and the seat of my pants I suggest ve begin vith the in vestigation and documentation of the analysis for the following assembly sequences;

1. $\frac{1}{03 L}$

2. $03 R$

3. O2L,O1L,O1R,O2R

4. OAI

5. $O R$

6. OSL

7. OSR

8. O6I

9. O6R

10. OTL

11. OTR

12. OBL

13. OBR

14. Mo

15. M1L

16. MIR

17. M2L

18 M2R

19. Y3L

20. $\mathrm{M}$ R

21. MLL

22. MR

23. ISI

24. $10 \mathrm{R}$

23. NIL

26. $\operatorname{man}$

27. $\mathbf{1 7}$

28. $47 R$

29. 18

30. III
B

03L

03R

O2L,OIL,O1R,ORR

Mo

MIL

MIR

MIL

MLR

M3L

M3R

MAL

MQR

MSL

MSR

M6L

M6R

M7L:

MTR :

48 .

II

OLI

OR

OSI

CSR

OSL

as

or

OrR

061

O8R c

$03 \mathrm{~L}$

03R

ORL,OLL,O1R,O2R

Mo

MIL

M1R

M2L

M2R

M3L

M3R

IH

M4L

MR

MSR

MSL

M6R

M6L

MTR

I7L

48

OL

OR

os?

osl

OSL

OSL

on.

OTR

o

oss

The modules are identifiod 0 for (outer numbered 1-s begining at the botton). 4 for middle (numbered O-s boginning at tho botton), I for ingor, and 2 for right and $L$ for boft of center as looking toward the detector center.

ICEF, IX,PR,RL,DU,DO,SR,KT,RT, 


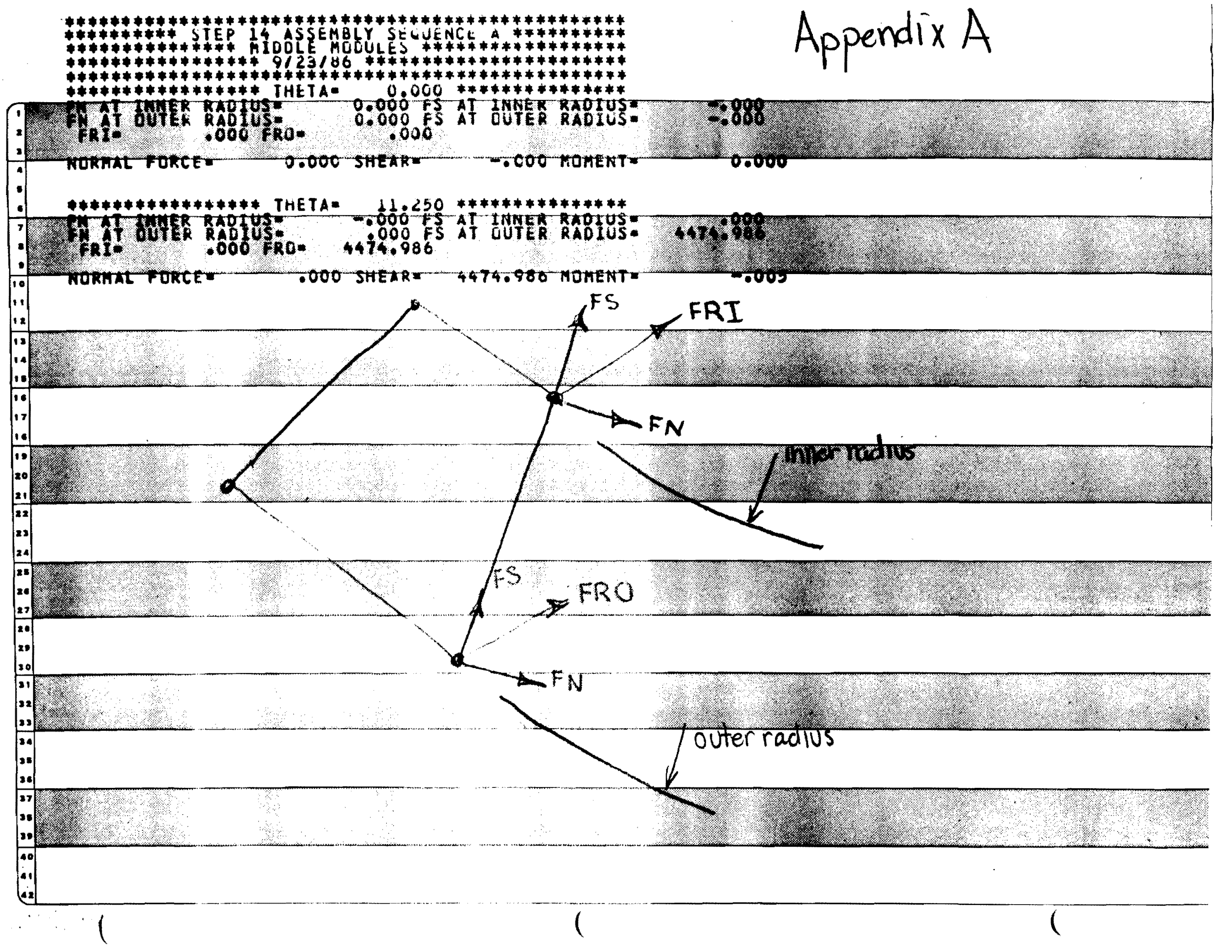




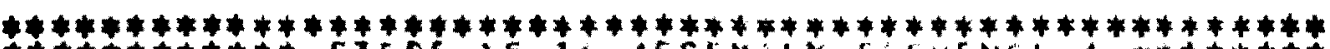

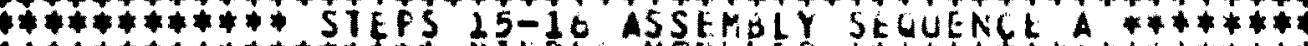

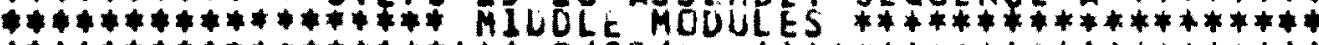

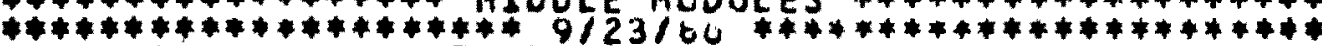

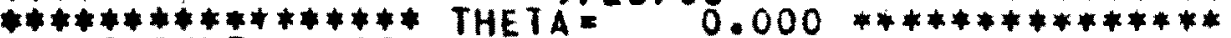

FN AT INNER RADIUS= 0.000 FS AI INNER RACIUS

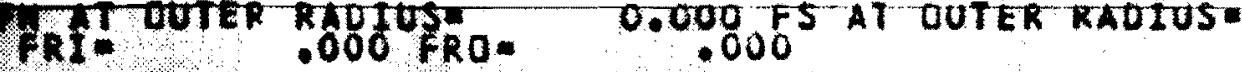

UORLAL FORCE $\quad 0.000$ SHEAR= .000 MOHENT

UOREAL FORCE $\quad 0.000$ SHEARE . .000 MONENT.

.000

.000

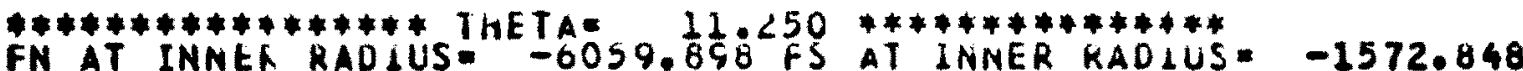

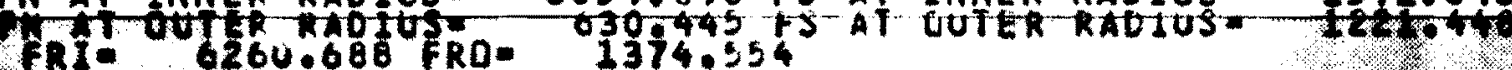

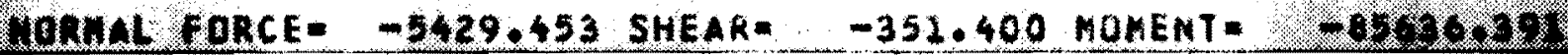

$* * * * * * * * * * * * * * * *$ THEIA $=33.750$ **************

FN AT INNER RADIUS = $-.000^{\circ}$ ES AI INNER KAOIUS -

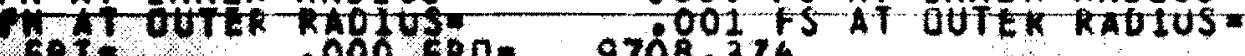

9700.000

Tron Lu FORCE

001 SWEAR=

9708.376 HOHENT

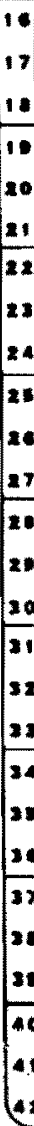

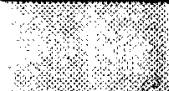

$-10$ 


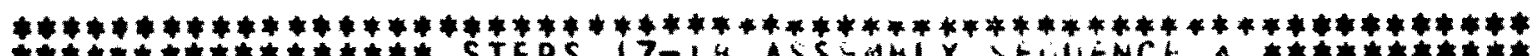

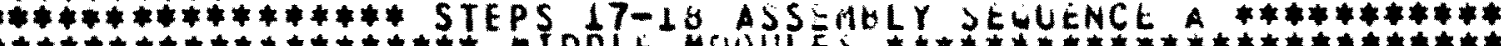

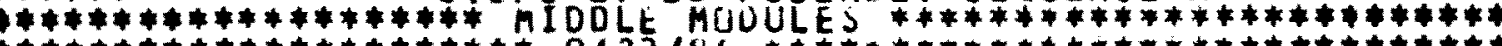

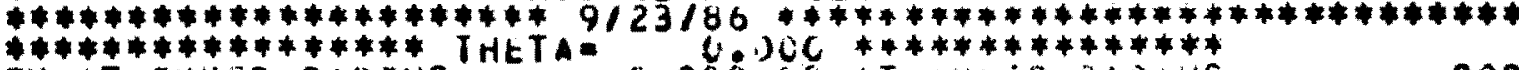

FN AT INNER RADIUS-

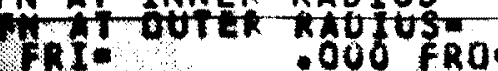

$0.000^{\circ}$ FS AT LNRER RAOTUS =

.000

Trual snirce.

0.000 SHEAR

. OOO MOMENT:

0.001:

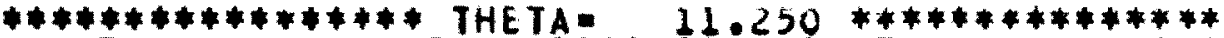

FN AT INNER RADIUS=-15521.2CO FS AT INNER KAOIUS- -1741.997

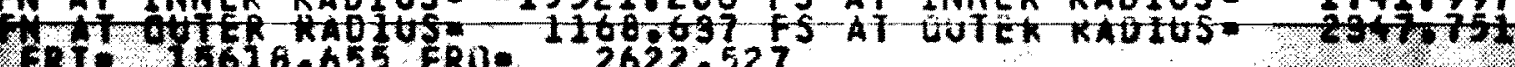

DONAL COACE -14352.569 SHEAR =

005.753 MOMENT $=-213029.00$

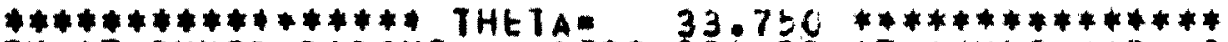

FN AT INNER RADIUS=-8720.096 FS AT INNER RAOLUS= -3471.988

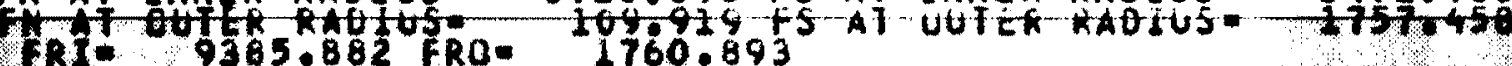

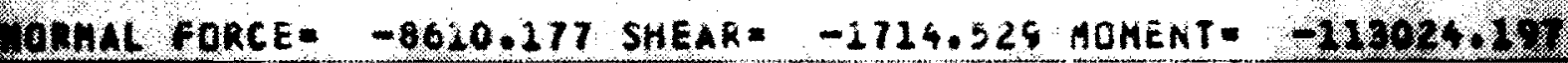

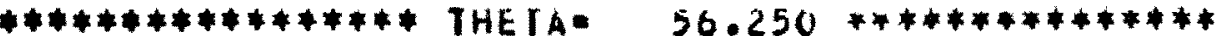

EN AT INNER RADIUS - $-.000^{\circ} \mathrm{FS}$ AT INNER KALIUS=

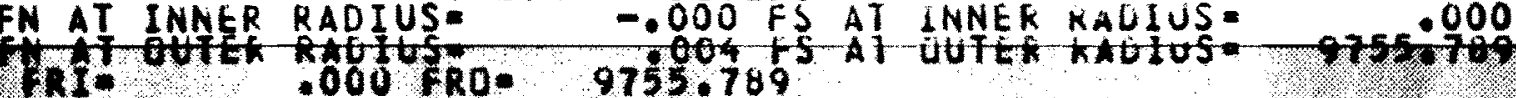

YONWL FonCE:

.004 SHEAR =

9755.789 AOMENT $=$

$-0.0$

\begin{tabular}{l}
22 \\
23 \\
24 \\
28 \\
20 \\
27 \\
20 \\
20 \\
20 \\
30 \\
21 \\
32 \\
32 \\
\hline 34 \\
38 \\
38 \\
\hline 23 \\
28 \\
20 \\
\hline 40 \\
401 \\
22 \\
\hline
\end{tabular} 


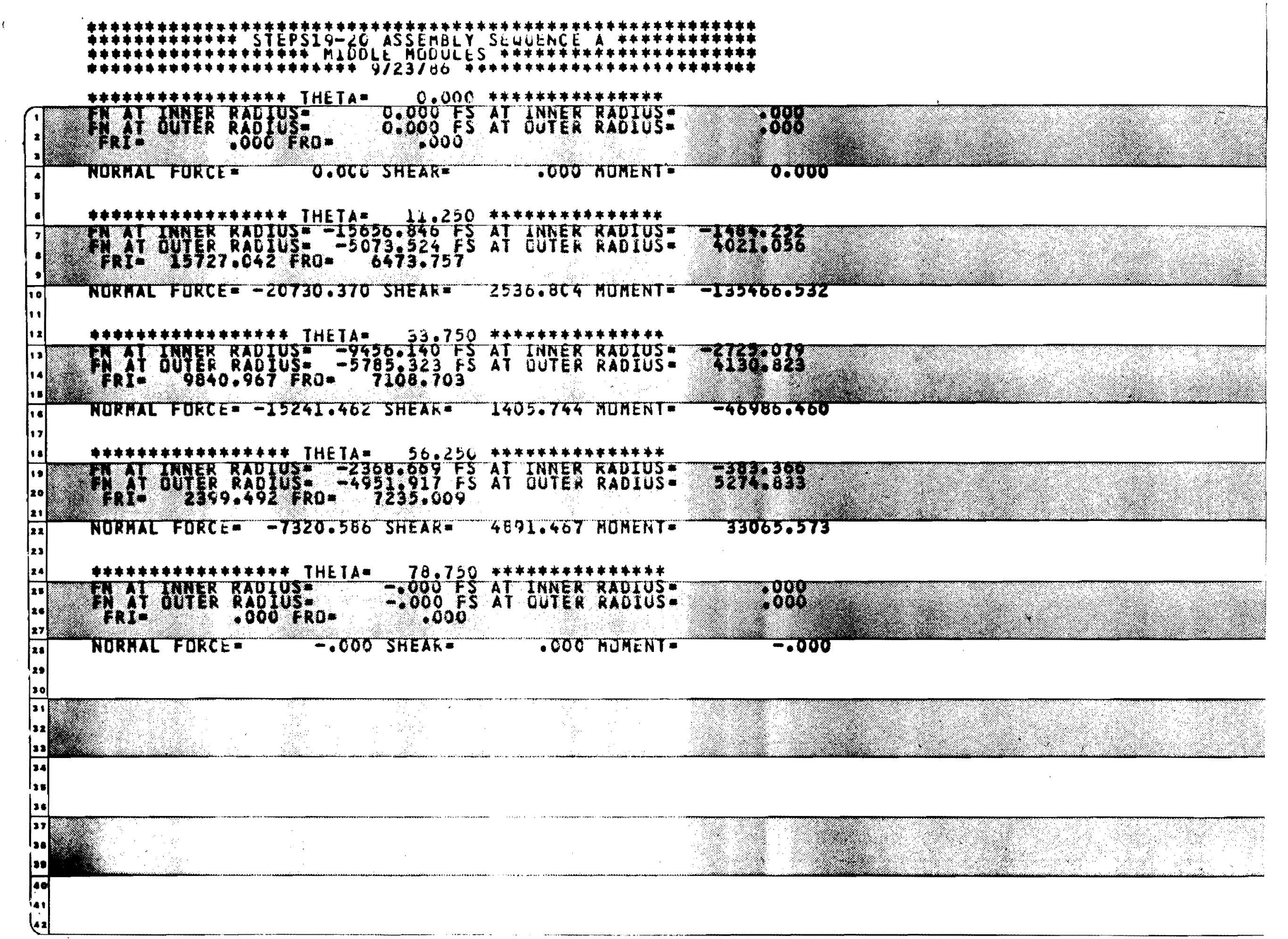




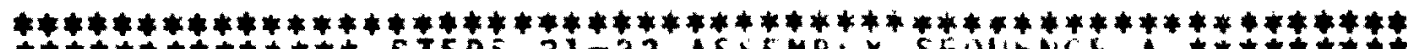

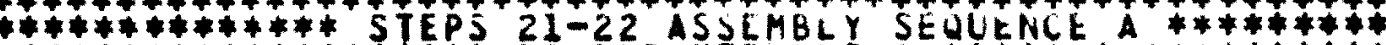

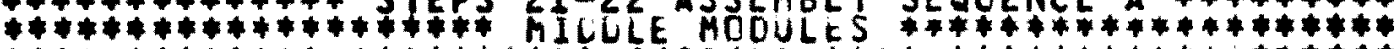

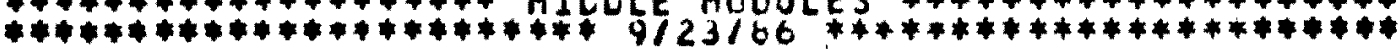

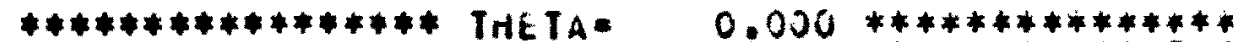

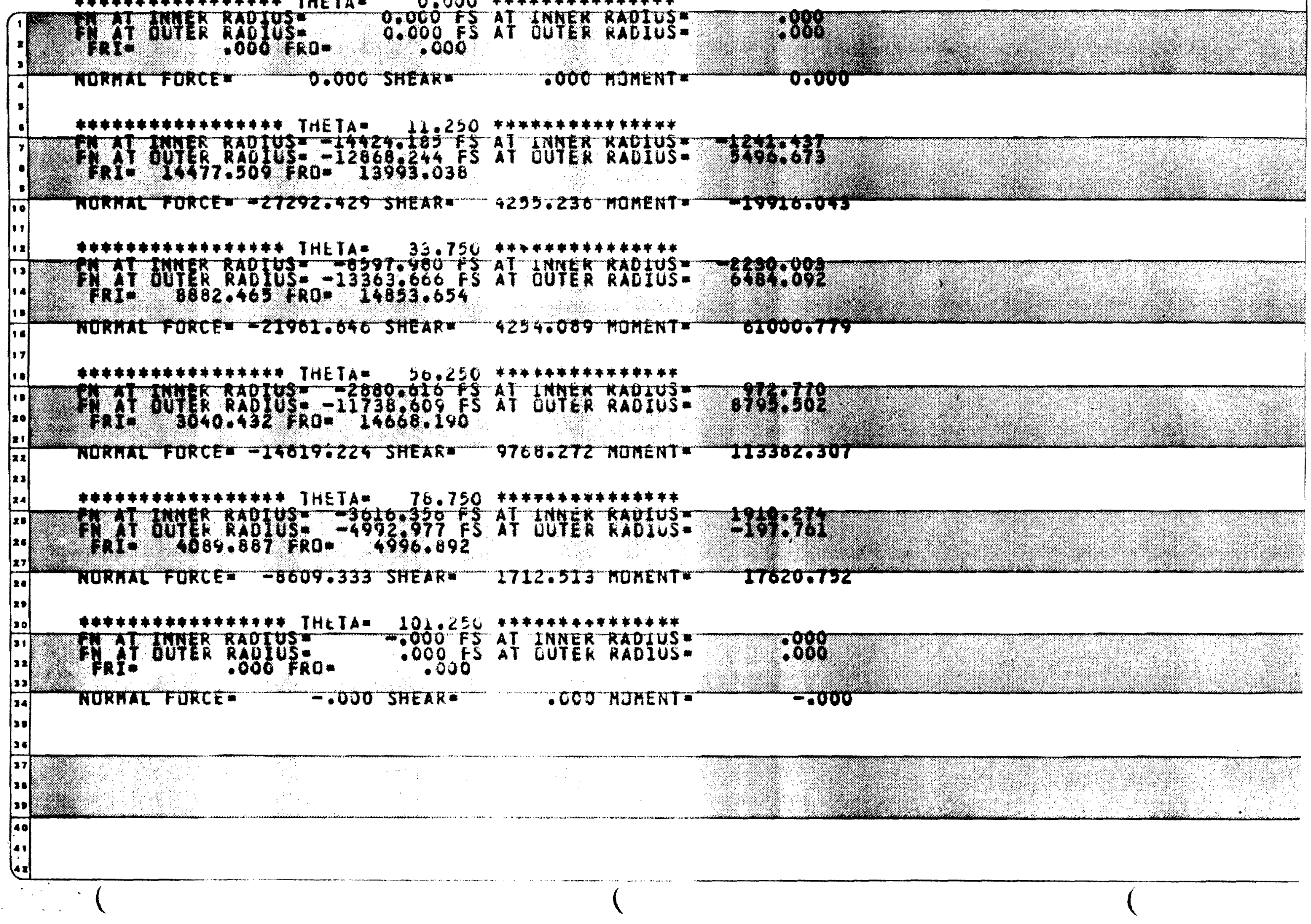




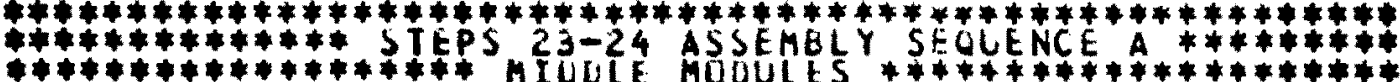

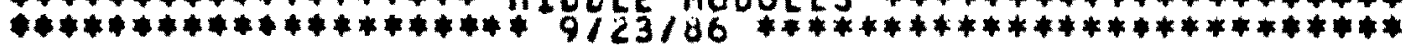

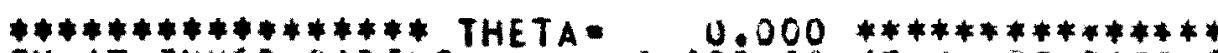

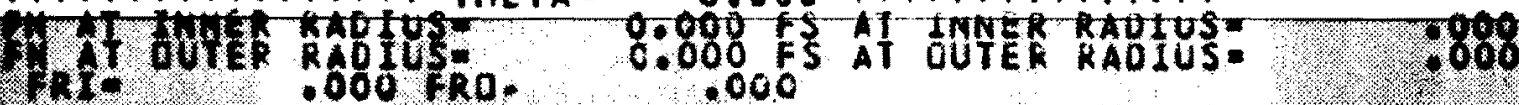

NOKWAL FOREE - 0.000 SHEAR - . .000 NOMENT

2.0 .000

$* * * * * * * * * * * * * * * *$ THETA $\quad 1+200 * * * * * * * * * * * * * *$

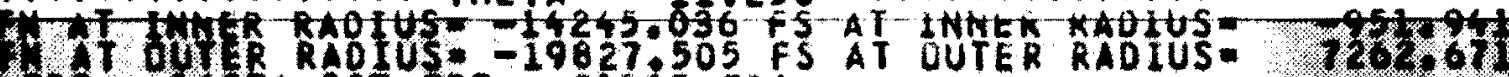

Rnt - 4276.807 FRO= 21115.784

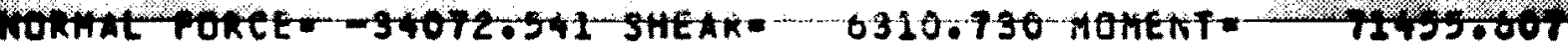

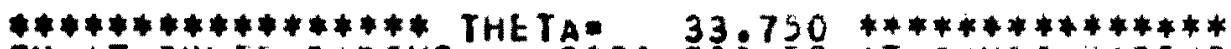

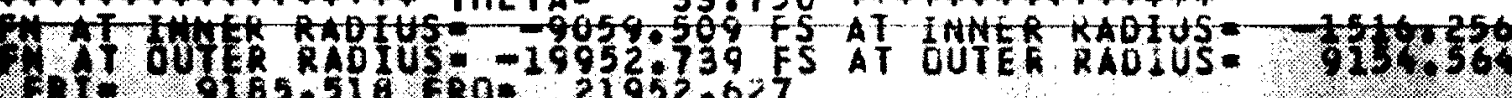

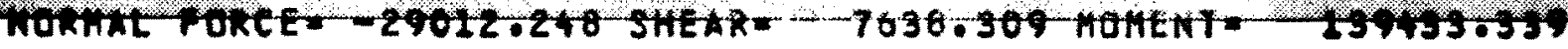

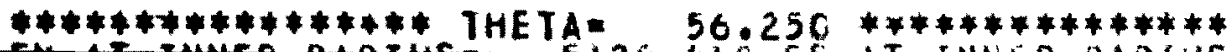

At 6 O

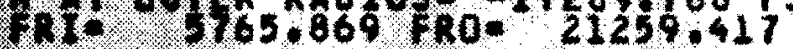

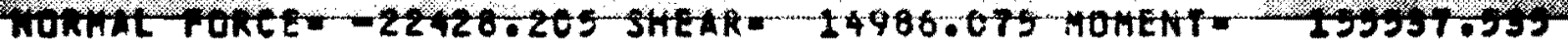

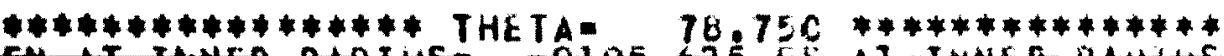

FRI DUP

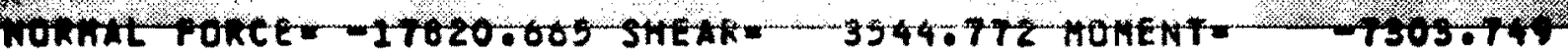

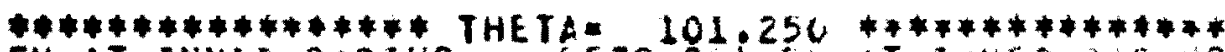

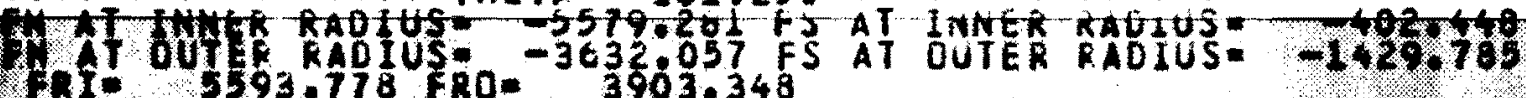

NORTKL FORCE -9211.338 SHEARE -1832.234 MOMENT -24924.471

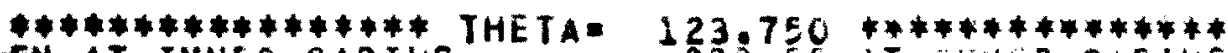

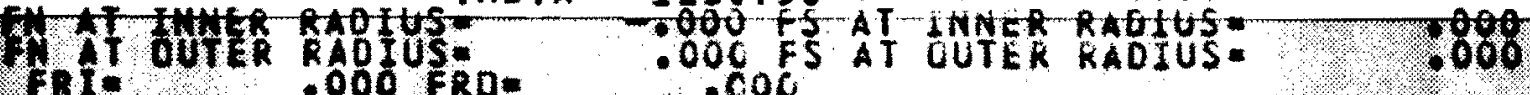

WORHAL POREE : .0OO SHEAR = ....... .OO MOHENT = $-.0000$ 


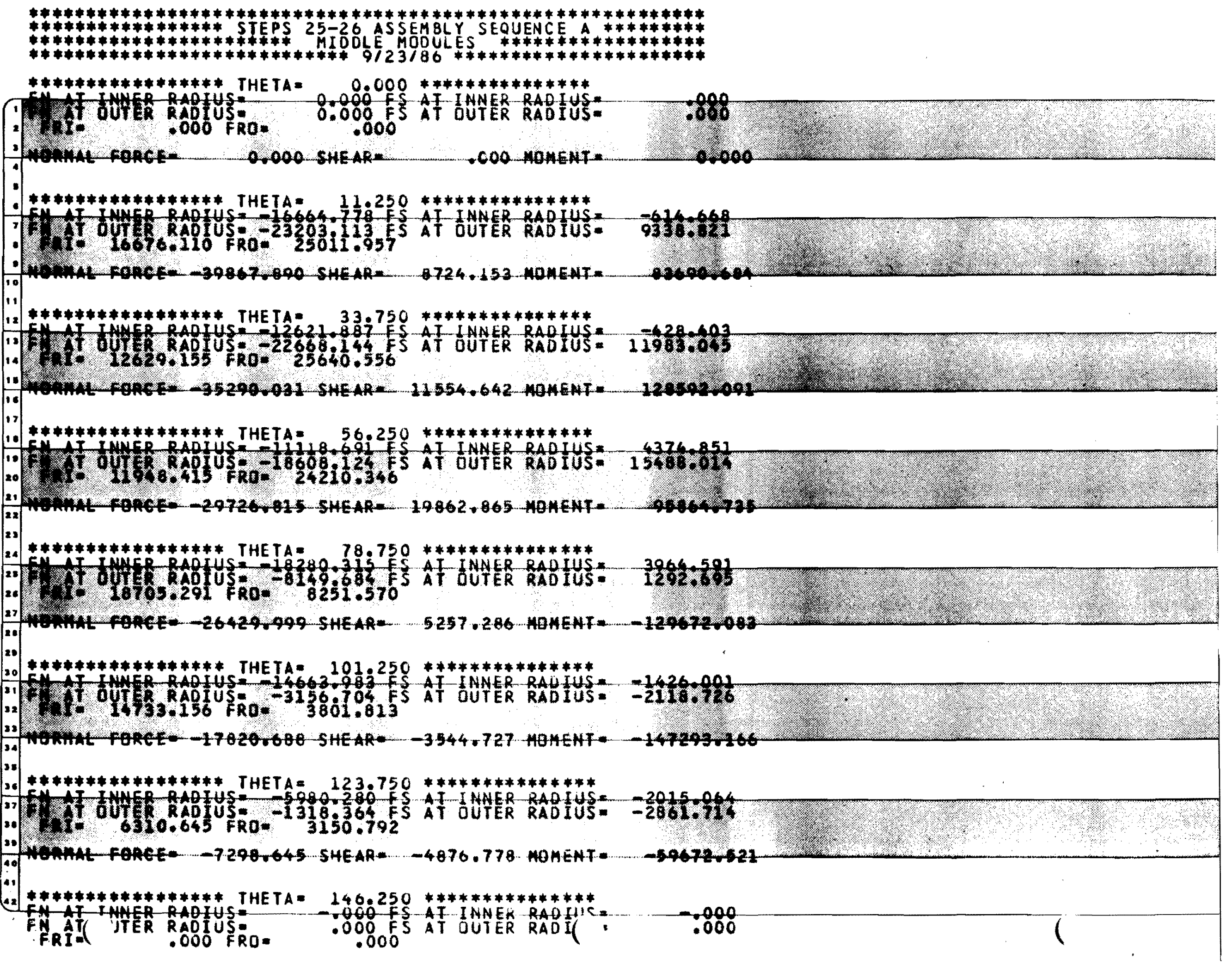




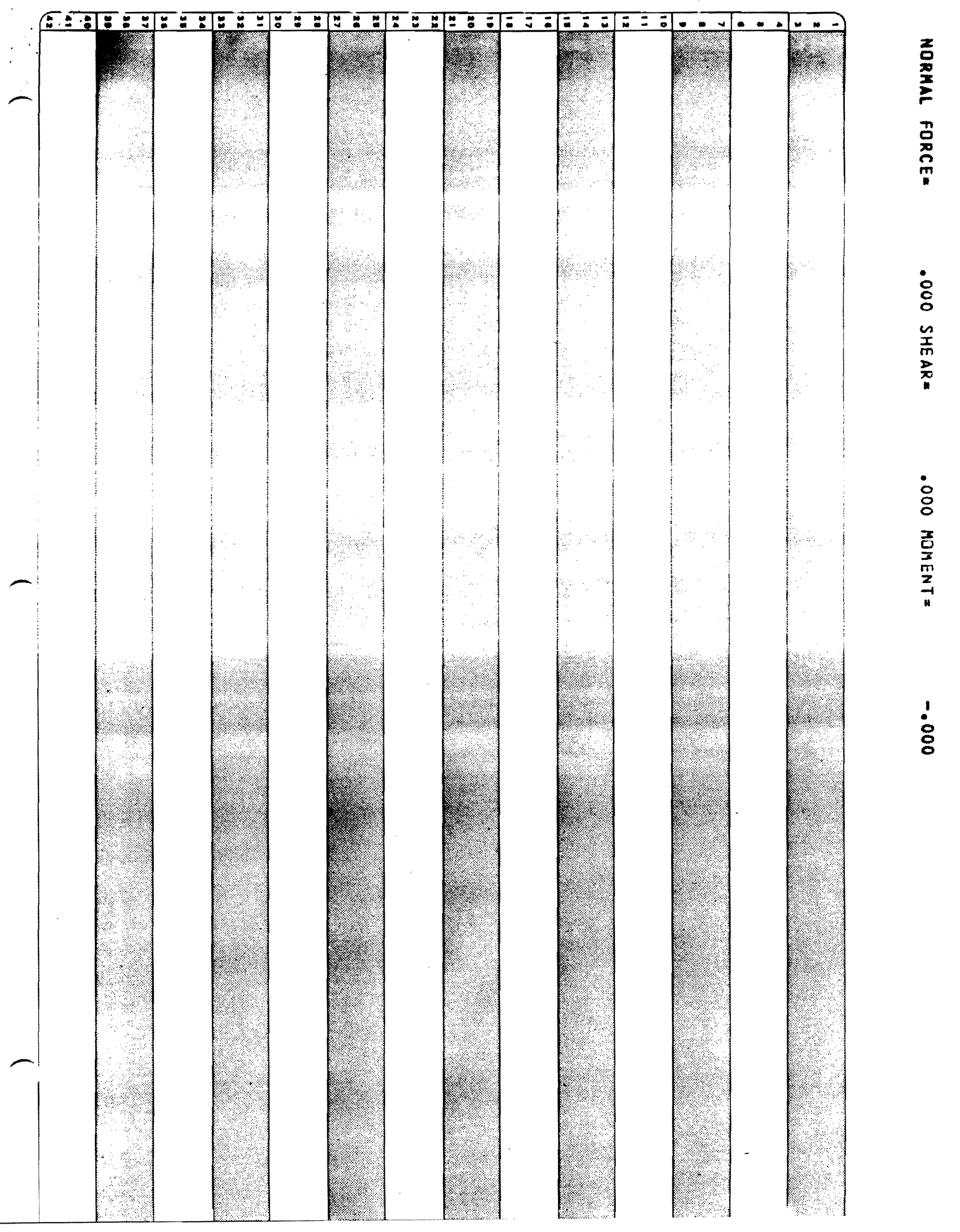




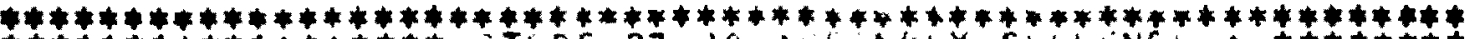

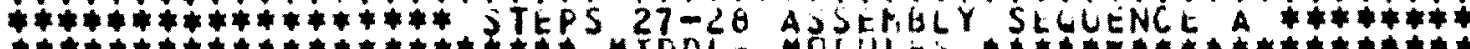

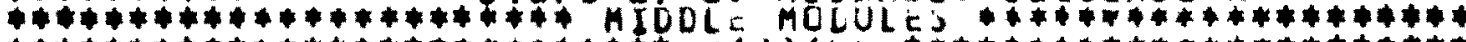

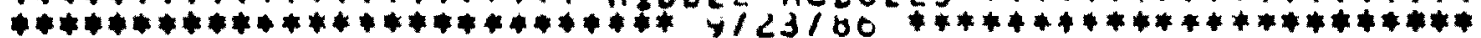

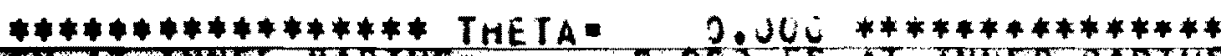

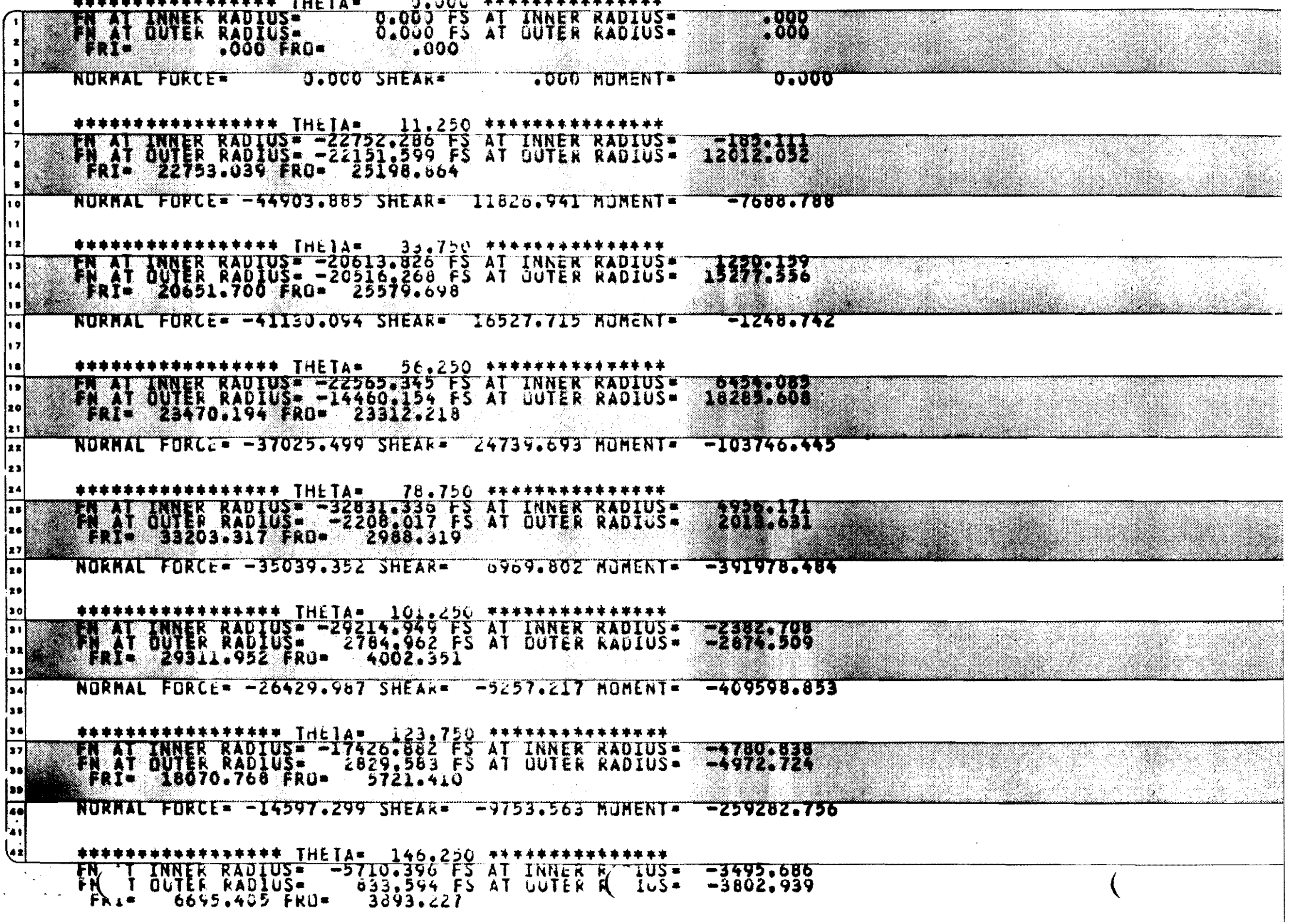


NORMAL FURCE $=-4870.803$ SHEAR $=-7248.624$ MUMENT $=-83763.073$

$-$

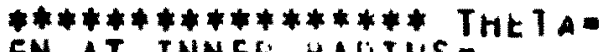
FN AT INAER KALIUS:
$166.700 * * * * * * * * * * * * * * *$ .000 FS AT INAER KALIUS: .000
GonkAL rosce: .000 FkU
.000 SHEAKE
.000 MONENT:
-.000
.000

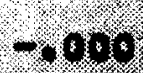

12, 10
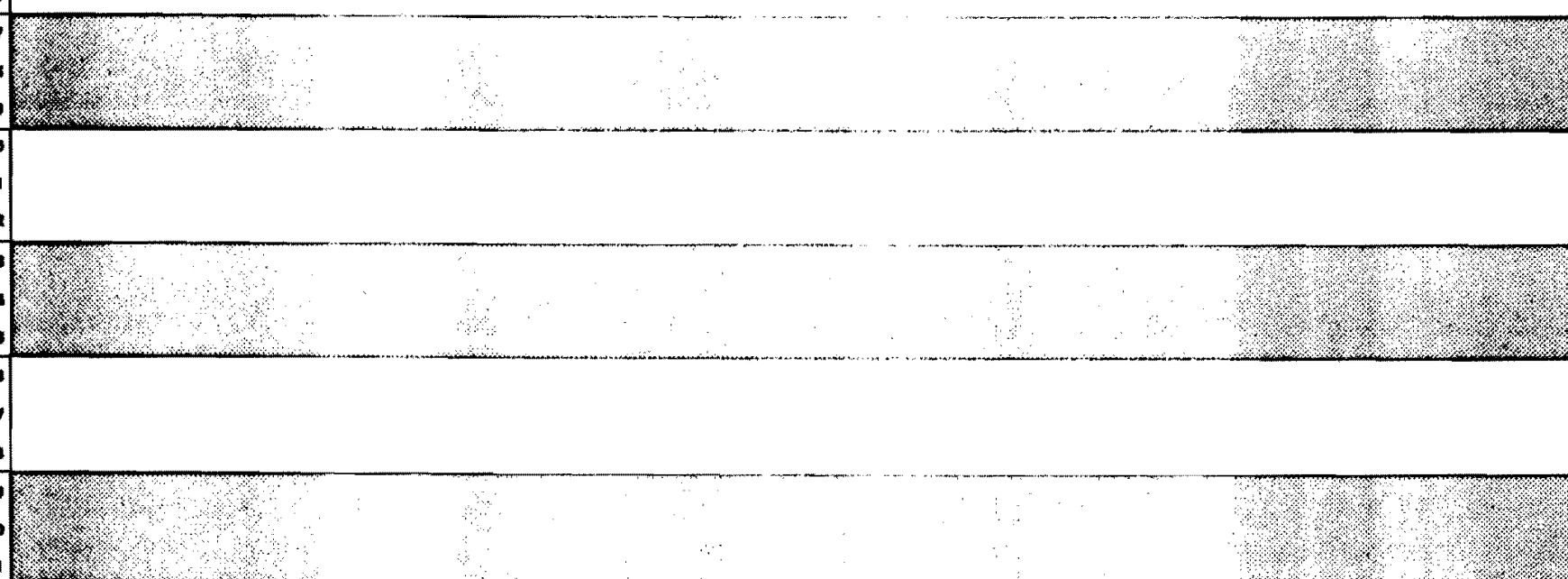

20.

20

30

31

32

3

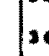

3

a 


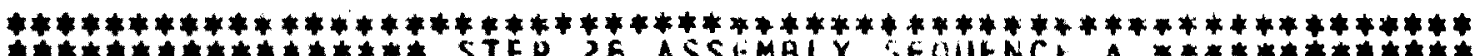

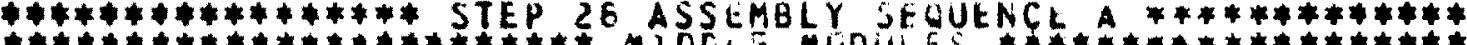

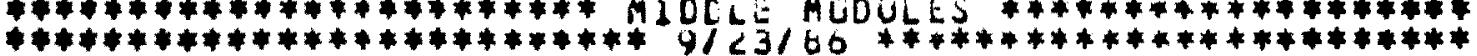

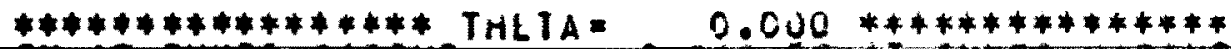

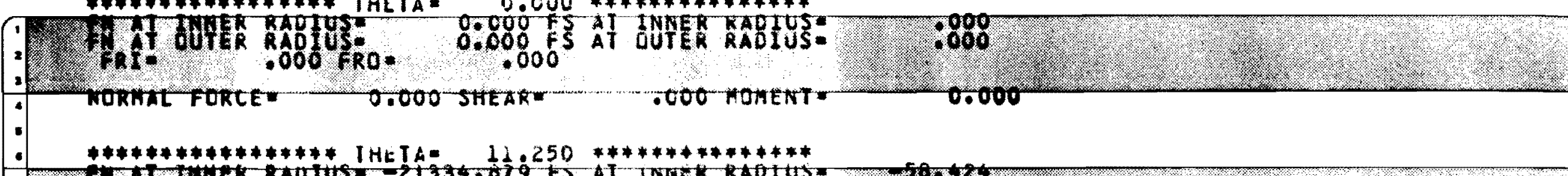

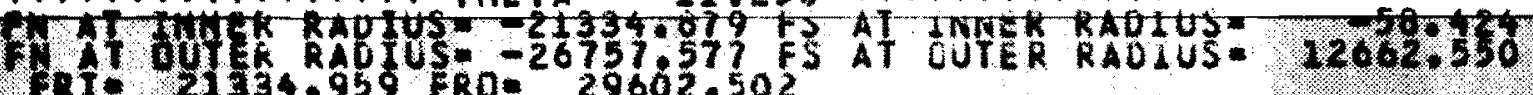

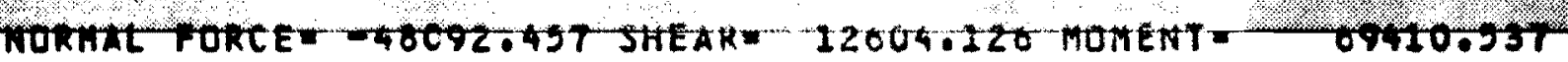

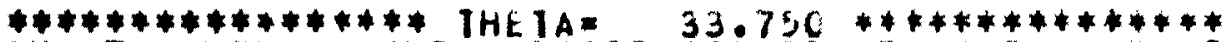

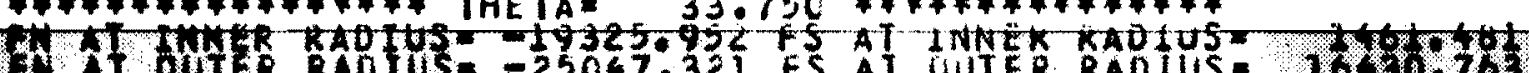

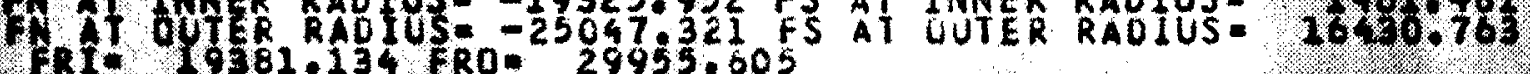

NORHAL FORCE -44373.273 SHEARE 37892.243 MOMENTE

73233.914

$* * * * * * * * * * * * * * * * *$ THETA= $50.250 * * * * * * * * * * * * * * *$

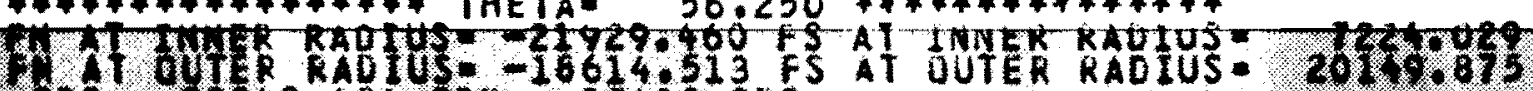

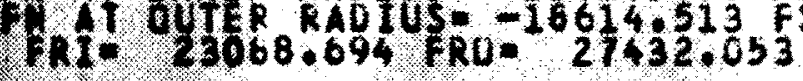

FURNAL FURCE -40543.973 SHEAR $=27373.904$ MONENT - -42431.319

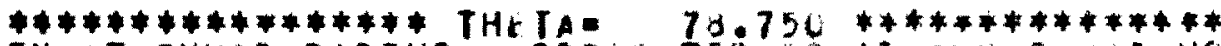

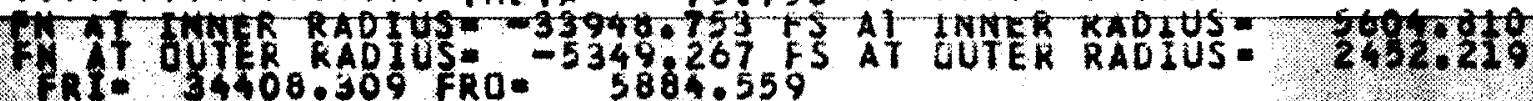

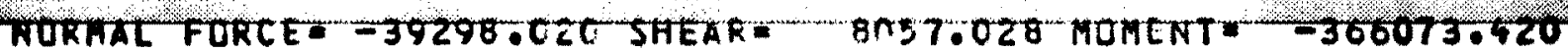

$* * * * * * * * * * * * * * * *$ THE IA $=10$ - $250 * * * * * * * * * * * * * *$

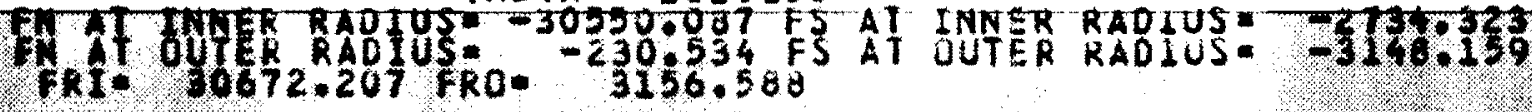

NORHAL FURCE $=30780.620$ SHEAR $=-5062.4$ BI MJHEIT $=-388090.274$

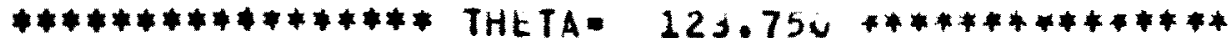

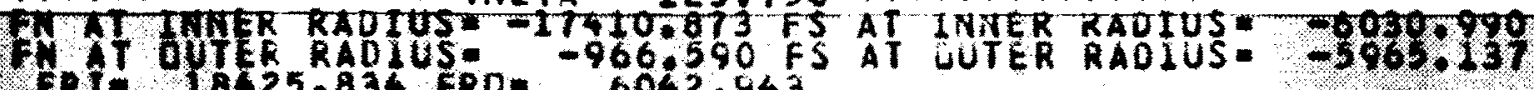
FRT- 18525.834 FRO 6042.943

FURWXT FORCE $=-18377.463$ SHEAR $=-1 \pm 595.128$ MUMENTW-210486.818

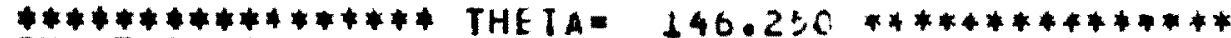

FA T INEE RATIUS -2980.194 FS AT INNER Fi TUS:-5450.619

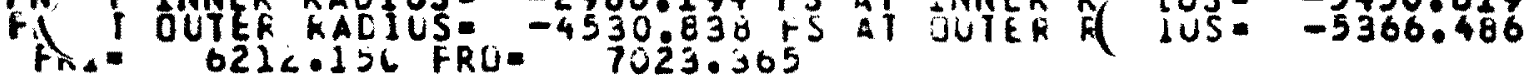


NURMAL FORLE $=-7511.03 \angle$ SHEAK $=-100.7 .165$ MUMENT $=19848.236$

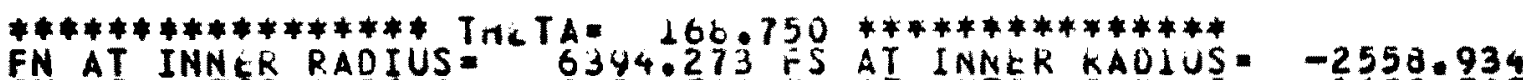

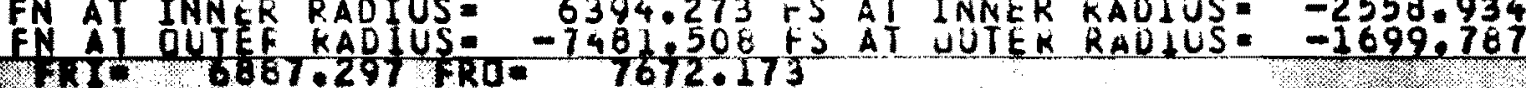

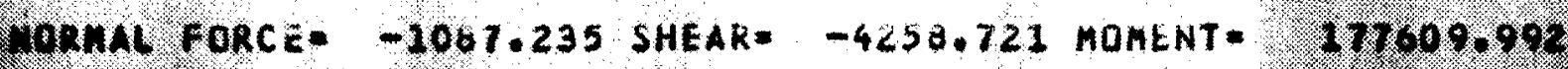

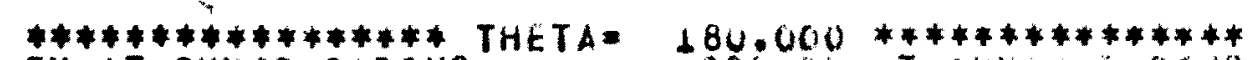

FN AT INNER RAOIUS: $\quad-.000$ F' AT INNEK RACLUS.

FN AT OUTER KALIUS:

.0UO IS AT UUTER KAOIUS:

TONHNL FORCE.

-.000 SHEAK=

-.000 MOMENT

-.000
.000

10

11

14

10

iv

1

20

\begin{tabular}{|l|}
21 \\
22 \\
21
\end{tabular}

22

24

$2=$

27

2o

20

30

2

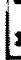

20

12

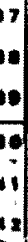

